\title{
Calidad de vida y percepción de apoyo social en personas con HIV en Bogotá, Colombia
}

\author{
José Moreno-Montoya ${ }^{1}$, Ana M. Barragán², Margin Martínez³, \\ Amanda Rodríguez ${ }^{4}$, Ángela Carmela González ${ }^{5}$
}

\author{
1 Grupo de Medicina Comunitaria, Universidad El Bosque, Bogotá, D.C., Colombia \\ 2 Escuela de Medicina y Ciencias de la Salud, Universidad del Rosario, Bogotá, D.C., Colombia \\ 3 Grupo de Investigación en Ciencias de la Rehabilitación, Escuela de Medicina y Ciencias de la Salud, \\ Universidad del Rosario, Bogotá, D.C., Colombia \\ 4 Instituto de la Familia, Universidad de la Sabana, Chía, Colombia \\ 5 Hospital Universitario Mayor Méderi, Bogotá, D.C., Colombia
}

Introducción. En estudios llevados a cabo en países desarrollados se ha establecido que las personas con HIV/sida refieren tener una menos calidad de vida y menor apoyo social.

Objetivo. Explorar la asociación entre la percepción del apoyo social afectivo o de personas de confianza y cada dimensión de la calidad de vida relacionada con la salud en personas con HIV/sida en Bogotá.

Materiales y métodos. Se hizo un estudio de corte transversal en personas con HIV/sida seleccionadas por conveniencia, en el marco de un programa de atención en una red hospitalaria de Bogotá. Se utilizaron los cuestionarios de calidad de vida relacionada con la salud SF36 y el cuestionario genérico de apoyo social funcional Duke-UNC-11. Se utilizaron modelos de regresión lineal en el análisis.

Resultados. Se evidenció una relación directa entre la dimensión del bienestar emocional de la calidad de vida, el apoyo social afectivo $\left(B=7,36 ; \mathrm{IC}_{95 \%}, 1,04-13,68\right)$ y el de personas de confianza $(B=11,63$; $\left.I C_{95 \%} 5,30-17,96\right)$, así como entre las dimensiones de la función física, el desempeño emocional y el dolor corporal y la percepción del apoyo social de tipo afectivo, y entre el apoyo social de personas de confianza y las dimensiones de la vitalidad y la función social. Se encontró una relación inversa entre los promedios de los puntajes de las dimensiones de desempeño emocional, desempeño físico y salud general y la percepción del apoyo social de tipo afectivo con la primera dimensión y el de personas de confianza con las dos últimas.

Conclusiones. Los sujetos con una mejor percepción del apoyo social reportaron una mejor calidad de vida relacionada con la salud, lo cual puede servir de base para la planeación, el diseño y la implementación de planes de atención médica que incorporen variables clínicas, paraclínicas y del entorno del paciente.

Palabras clave: HIV; calidad de vida; apoyo social; modelos lineales; percepción; Colombia. doi: https://doi.org/10.7705/biomedica.v38i4.3819

\section{Quality of life and perceived social support in people with HIV in Bogotá, Colombia}

Introduction: People living with HIV/AIDS in developing countries tend to have a lower quality of life and less social support compared with those in developed countries.

Objective: To explore the association between affective social support or social support generating confidence and each dimension of the quality of life related to health among people with HIV/AIDS from Bogotá.

Materials and methods: We conducted a cross-sectional study of people living with HIV/AIDS using convenience sampling of a care program in a hospital network in Bogotá. The quality of life

\section{Contribución de los autores:}

José Moreno-Montoya: plan de análisis

Ana M. Barragán: protocolo del proyecto y plan de análisis

Margin Martínez: diseño y concepción del protocolo, recolección de la información

Amanda Rodríguez: diseño del estudio, recolección de la información e interpretación de los datos

Ángela Carmela González: recolección de la información y comunicación con pacientes

Todos los autores participaron en la escritura del manuscrito. 
questionnaire SF36 and the generic social support questionnaire Duke-UNC-11 were used, along with linear regression models for the analyses.

Results: There was a direct relationship between the emotional well-being dimension of quality of life and the social support systems of affectivity $(B=7.36 ; 95 \% \mathrm{Cl}: 1.04 ; 13.68)$ and those generating confidence ( $B=11.63 ; 95 \% \mathrm{Cl}: 5.30 ; 17.96)$. There was a correlation between the dimensions of physical function, emotional performance, pain, and perceived affective social support. Likewise, we detected relations between the perception of social support generating confidence and the dimensions of vitality and social function. Contrarily, we found an inverse relationship between the averages of the dimension of emotional performance and the perceived affective social support, as well as between the dimension of physical performance and general health with social support generating confidence.

Conclusions: Subjects with higher levels of social support had higher levels of quality of life related to health. This finding offers an opportunity for the design and implementation of healthcare plans that incorporate clinical, para-clinical and environmental variables of the patient.

Key words: HIV; quality of life; social support; linear models; perception; Colombia. doi: https://doi.org/10.7705/biomedica.v38i4.3819

La infección por el virus de la inmunodeficiencia humana (HIV) y el síndrome de inmunodeficiencia adquirida (sida), afectan tanto la condición física de los pacientes como su desempeño social, su salud mental y su bienestar financiero (1).

Desde el inicio de la pandemia, a principios de la década de los 80, la enfermedad constituyó una de las mayores preocupaciones en salud pública a nivel mundial, con cerca de 80 millones de personas infectadas para finales de 2013 y 35 millones con la enfermedad (2). Es frecuente que esta población reporte ser víctima de estigma y discriminación, situaciones que afectan su calidad de vida $(3,4)$, principalmente en cuanto a sus niveles de bienestar emocional (5), lo cual se asocia con la insatisfacción de las necesidades que se derivan de la interacción con los demás: filiación, afecto, pertenencia, identidad, seguridad y aprobación $(4,6)$.

A menudo, estas personas se ven afectadas por sentimientos de aflicción y desesperanza y, en algunos casos, por la ideación y la planeación suicidas (7). Es habitual que recurran a estrategias de afrontamiento basadas en la evitación, lo que a su vez se asocia con trastornos mayores del humor (depresión y ansiedad) y con disminución de la vitalidad (8), estados que conducen al menoscabo del desempeño social del individuo y a la disminución de la magnitud y la frecuencia de las actividades en su entorno social (9).

\section{Correspondencia:}

Ana María Barragán, Escuela de Medicina y Ciencias de la Salud, Universidad del Rosario, Carrera 24 № 63C-69,

Bogotá, D.C., Colombia

Teléfono: (571) 297 0200, extensión 3408

ana.barragan@urosario.edu.co
Los resultados de algunos estudios en países en desarrollo han sido contradictorios en cuanto a los efectos en la dimensión física de la calidad de vida, especialmente en aquellos hombres que perciben poco apoyo social $(5,10)$. Por otra parte, se ha sugerido que la percepción de un apoyo social limitado constituye una barrera para la prestación eficaz de los servicios de salud (4), así como para el cumplimiento de la terapia antirretroviral (11).

Debe reconocerse, entonces, que hay un rezago en el apoyo social como modulador de los efectos de acontecimientos vitales estresantes o degenerativos de la salud (12). Si bien esta situación ha sido ampliamente discutida $(13,14)$, persisten serias dificultades metodológicas para medir el apoyo social $(12,14,15)$; además, la exigüidad de publicaciones científicas sobre poblaciones provenientes de países en desarrollo limita las conclusiones sobre el tema (10). En el caso de Colombia, las estimaciones recientes señalan que en el 2014 había cerca de 145.000 personas con HIV/sida y en el 2012 se reportaron 6.500 muertes, aunque no se conoce la proporción de casos debidos a comportamientos sexuales de riesgo 0 al uso inadecuado de elementos hematológicos (16).

El objetivo del presente estudio fue responder a las siguientes preguntas: a) ¿existe asociación entre la percepción del apoyo social afectivo o el apoyo social de personas de confianza y cada una de las dimensiones de la calidad de vida relacionadas con la salud? y b) ¿cuál es la magnitud del cambio en la calidad de vida relacionado con el apoyo social percibido? Las dos preguntas se plantearon para la población adscrita a un programa de atención integral ambulatoria en Bogotá. 


\section{Materiales y métodos Diseño}

Se hizo un estudio de corte transversal en personas con HIV/sida atendidas en la Red Hospitalaria Méderi en Bogotá. La red está compuesta por un hospital de cuarto nivel de complejidad y uno de segundo nivel, que atienden a la que se conoce como la cohorte más antigua de pacientes con este diagnóstico en el marco del programa B24, el cual coordina la atención integral e interdisciplinaria para dichas personas y está conformado por 17 profesionales de las áreas de infectología, medicina general, asistencia social, nutrición, psicología, química farmacéutica, enfermería, medicina familiar, y por personal administrativo, que orientan a los pacientes mediante estrategias de prevención de enfermedades y complicaciones, y además, dirigen la asistencia ambulatoria (17).

Se consideraron como elegibles todas las personas mayores de 18 años que pertenecieran al programa y no tuvieran alteraciones en sus habilidades comunicativas ni discapacidad cognitiva (18). Se seleccionó una muestra no probabilística entre las personas con $\mathrm{HIV} /$ sida atendidas en consulta de medicina general, nutrición, enfermería, psicología o infectología en las instituciones de la red durante el periodo comprendido entre mayo de 2014 y septiembre de 2015. En cada consulta se explicó el objetivo del proyecto y las personas que aceptaron firmar el consentimiento informado contestaron las preguntas de un cuestionario hechas por encuestadores entrenados.

El protocolo del estudio fue aprobado por el Comité de Ética de la Universidad del Rosario de Bogotá y por el Comité Técnico-Científico de la red Méderi. En el momento de este estudio, hacían parte del programa cerca de 960 personas con HIV/sida con edades entre los 7 y los 84 años, en su mayoría hombres (84\%), aunque para el estudio solo se consideraron los mayores de edad.

\section{Variables e instrumentos}

Las variables demográficas se evaluaron mediante una encuesta diseñada para este estudio que incluía la edad categorizada en grupos de 18 a 30 años y de 45 o más, así como el sexo, el nivel educativo (no profesional y profesional), la residencia en Bogotá(sí/no), el estrato socioeconómico (bajo, medio o alto), la orientación sexual (heterosexual, homosexual u otra), el estado civil (soltero, casado, unión libre, divorciado o viudo), la ocupación (desempleado, estudiante, trabajador informal, jubilado o independiente).

Para medir la calidad de vida relacionada con la salud, se utilizó el cuestionario SF36 en español, previamente validado en población colombiana con HIV/sida (3). Este cuestionario evalúa la calidad de vida relacionada con la salud percibida por el sujeto en las últimas cuatro semanas a partir de 36 temas que se agrupan en ocho dominios: dolor corporal, desempeño emocional, desempeño físico, función física, función social, salud general, salud mental y vitalidad. El sistema de puntuación fue el mismo utilizado en estudios previos (3).

En cuanto al apoyo social percibido, se utilizó el cuestionario genérico de apoyo social funcional Duke-UNC-11 en español, aún no validado en población colombiana (19), el cual consta de 11 preguntas con cinco opciones de respuesta que van de 1 (mucho menos de lo que deseo o quiero) a 5 (tanto como deseo o quiero). El instrumento evalúa dos dominios: el apoyo social afectivo y el apoyo social de personas de confianza. Las puntuaciones mínima y máxima por dimensión oscilan entre 6 y 30 puntos para el primero, y entre 5 y 25 para el segundo; se considera que existe apoyo social afectivo si se obtiene un mínimo de 18 puntos y, apoyo de personas de confianza, si se obtiene un mínimo de 15 puntos $(19,20)$.

\section{Análisis estadístico}

Para calcular la puntuación total de la calidad de vida, se usó el promedio de los valores obtenidos en el cuestionario SF36 y el puntaje por dimensión, como se ha reportado en otras publicaciones $(21,22)$. En el caso del cuestionario DUKE, se sumó el valor de la respuestas dadas en cada escala, como se ha reportado en estudios anteriores $(6,19)$.

El análisis exploratorio de las variables categóricas se hizo mediante el cálculo de medidas descriptivas de frecuencia y, el de las variables cuantitativas, mediante medidas de tendencia central y dispersión (media y desviación estándar, DE). Las comparaciones simples se hicieron con la prueba t de Student y los promedios de cada dimensión se ajustaron mediante modelos de regresión lineal (23). La elección del modelo final se basó en el aporte de cada variable según los lineamientos de la metodología de selección por pasos (24). Los modelos se validaron con respecto a sus supuestos estadísticos, incluidas la apreciación gráfica de los residuos y la prueba de homocedasticidad de Breush-Pagan (25). 


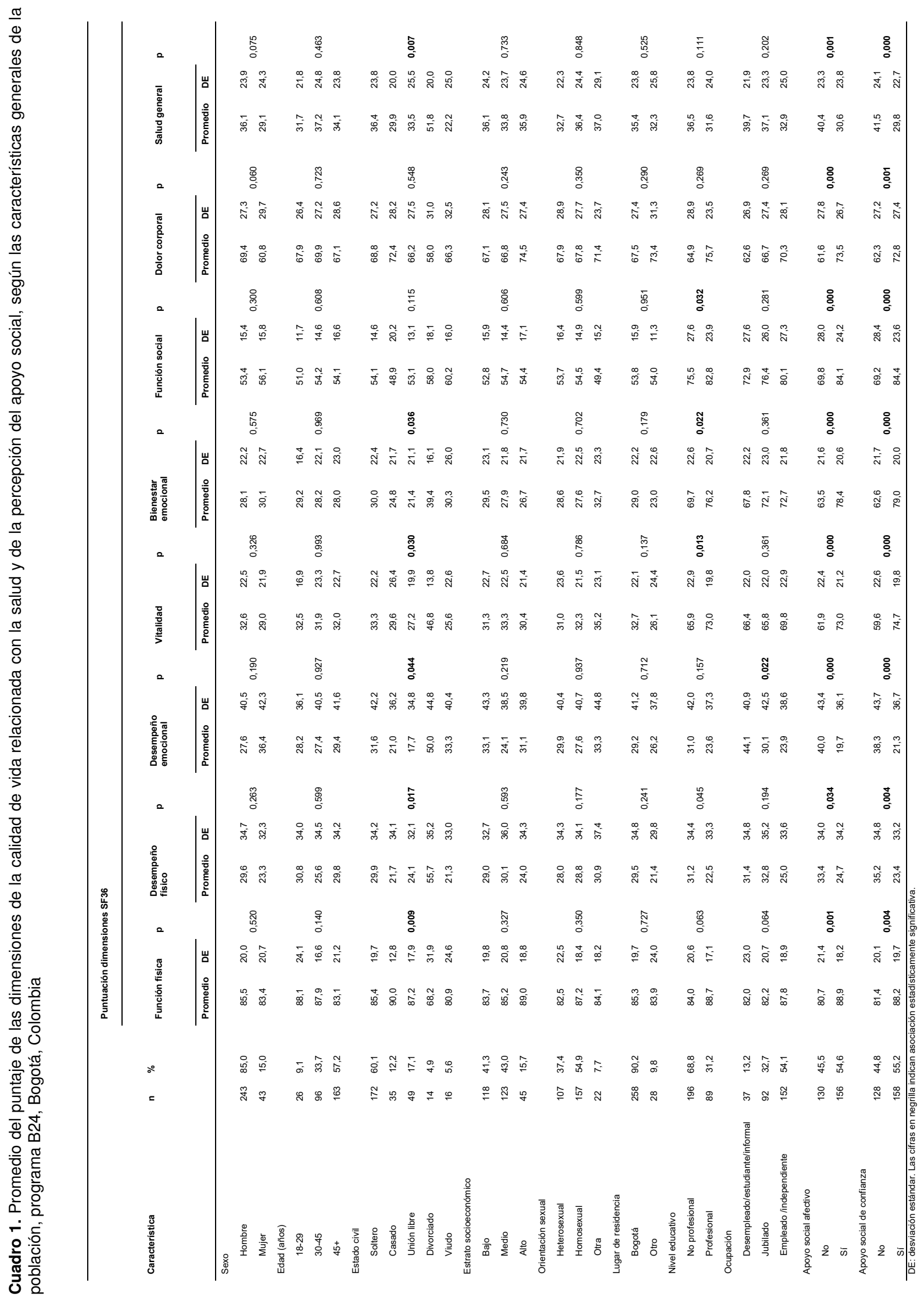


Cuadro 2. Cambio ajustado por el promedio en el puntaje de las dimensiones del cuestionario sobre calidad de vida relacionada con la salud SF36 en personas que viven con HIV/sida inscritas en el programa B24, Bogotá, Colombia, 2015

\begin{tabular}{|c|c|c|c|c|}
\hline \multirow{2}{*}{$\begin{array}{l}\text { Dimensiones } \\
\text { Función física }\end{array}$} & \multirow[t]{2}{*}{ Coeficiente } & \multirow[t]{2}{*}{$p$} & \multicolumn{2}{|c|}{$\left(\mathrm{IC}_{95 \%}\right)$} \\
\hline & & & & \\
\hline \multicolumn{5}{|l|}{ Orientación sexual } \\
\hline Homosexual Vs. heterosexual & 5,88 & 0,04 & 0,31 & 11,46 \\
\hline Otro Vs. heterosexual & 6,25 & 0,19 & $-3,03$ & 15,54 \\
\hline \multicolumn{5}{|l|}{ Estado civil } \\
\hline Casado Vs. soltero & 8,00 & 0,05 & 0,03 & 15,97 \\
\hline Unión libre Vs. soltero & 1,10 & 0,73 & $-5,25$ & 7,45 \\
\hline Divorciado Vs. soltero & $-14,73$ & 0,01 & $-25,83$ & $-3,64$ \\
\hline Viudo Vs. soltero & $-1,27$ & 0,81 & $-11,64$ & 9,10 \\
\hline Apoyo social afectivo (Sí Vs. No) & 8,11 & 0,00 & 70,96 & 82,41 \\
\hline \multicolumn{5}{|l|}{ Desempeño físico } \\
\hline \multicolumn{5}{|l|}{ Estado civil } \\
\hline Casado Vs. soltero & $-9,08$ & 0,15 & $-21,32$ & 3,16 \\
\hline Unión libre Vs. soltero & $-4,67$ & 0,39 & $-15,34$ & 6,01 \\
\hline Divorciado Vs. soltero & 23,81 & 0,01 & 5,57 & 42,06 \\
\hline Viudo Vs. soltero & $-9,17$ & 0,29 & $-26,30$ & 7,95 \\
\hline Escolaridad (profesional Vs. no profesional) & $-8,62$ & 0,05 & $-17,07$ & $-0,17$ \\
\hline Apoyo social de personas de confianza (Sí Vs. No) & $-10,37$ & 0,01 & $-18,24$ & $-2,50$ \\
\hline \multicolumn{5}{|l|}{ Desepeño emocional } \\
\hline Apoyo social afectivo (Sí Vs. No) & $-20,34$ & 0,00 & $-29,60$ & $-11,09$ \\
\hline \multicolumn{5}{|l|}{ Vitalidad } \\
\hline \multicolumn{5}{|l|}{ Estado civil } \\
\hline Casado Vs. soltero & 3,21 & 0,41 & $-4,43$ & 10,84 \\
\hline Unión libre Vs. soltero & 4,03 & 0,24 & $-2,66$ & 10,73 \\
\hline Divorciado Vs. soltero & $-11,91$ & 0,04 & $-23,36$ & $-0,46$ \\
\hline Viudo Vs. soltero & 9,12 & 0,10 & $-1,65$ & 19,88 \\
\hline Apoyo social de personas de confianza (Sí Vs. No) & 14,68 & 0,00 & 9,75 & 19,62 \\
\hline \multicolumn{5}{|l|}{ Bienestar emocional } \\
\hline Escolaridad (profesional Vs. no profesional) & 5,81 & 0,03 & 0,66 & 10,95 \\
\hline Apoyo social afectivo (Sí Vs. No) & 7,36 & 0,02 & 1,04 & 13,68 \\
\hline Apoyo social de personas de confianza (Sí Vs. No) & 11,63 & 0,00 & 5,30 & 17,96 \\
\hline \multicolumn{5}{|l|}{ Función social } \\
\hline \multicolumn{5}{|l|}{ Estado civil } \\
\hline Casado Vs. soltero & 6,20 & 0,19 & $-3,17$ & 15,57 \\
\hline Unión libre Vs. soltero & 10,34 & 0,01 & 2,11 & 18,56 \\
\hline Divorciado Vs. soltero & $-5,87$ & 0,41 & $-19,93$ & 8,19 \\
\hline Viudo Vs. soltero & $-2,29$ & 0,73 & $-15,51$ & 10,93 \\
\hline Escolaridad (profesional Vs. no profesional) & 7,69 & 0,02 & 1,26 & 14,12 \\
\hline Apoyo social de personas de confianza (Sí Vs. No) & 14,04 & 0,00 & 7,98 & 20,10 \\
\hline \multicolumn{5}{|l|}{ Dolor corporal } \\
\hline Escolaridad (profesional Vs. no profesional) & 10,18 & 0,00 & 3,43 & 16,93 \\
\hline Apoyo social afectivo (Sí Vs. No) & 11,12 & 0,00 & 4,84 & 17,40 \\
\hline \multicolumn{5}{|l|}{ Salud general } \\
\hline \multicolumn{5}{|l|}{ Estado civil } \\
\hline Casado Vs. soltero & $-6,14$ & 0,15 & $-14,51$ & 2,22 \\
\hline Unión libre Vs. soltero & $-1,35$ & 0,72 & $-8,70$ & 5,99 \\
\hline Divorciado Vs. soltero & 14,13 & 0,03 & 1,58 & 26,68 \\
\hline Viudo Vs. soltero & $-15,36$ & 0,01 & $-27,17$ & $-3,56$ \\
\hline Apoyo social de personas de confianza (Sí Vs. No) & $-11,56$ & 0,00 & $-16,97$ & $-6,15$ \\
\hline
\end{tabular}




\section{Resultados}

De los 286 pacientes, 43 (15,03 \%) eran mujeres. La mayor parte $(90,21 \%)$ de los sujetos incluidos en la muestra provenía de Bogotá; en cuanto a la edad, $9,12 \%$ de los sujetos tenía entre 18 y 29 años, en tanto que 33,68\% estaba entre los $30 \mathrm{y}$ los 45 años de edad.

El $54 \%\left(\right.$ IC $\left._{95 \%} 48,7-60,3\right)$ de los encuestados percibió apoyo social afectivo, mientras que el apoyo social de personas de confianza fue percibido por el $55 \%\left(\mathrm{IC}_{95 \%} 49,4-60,9\right)$ de los casos.

Los promedios de los puntajes y la distribución por sexo, edad, estado civil, estrato socioeconómico, orientación sexual, residencia en Bogotá (sí/ no), nivel educativo y ocupación, así como su distribución por presencia o ausencia de apoyo afectivo o de apoyo de personas de confianza en cada una de las dimensiones de la calidad de vida, se presentan en el cuadro 1.

En el análisis ajustado, se encontró una relación directa entre el apoyo social afectivo y aquel de personas de confianza, y mejores promedios en los puntajes de la dimensión del bienestar emocional. Asimismo, se encontró una relación directa entre el promedio de los puntajes en las dimensiones de la función física y el dolor corporal relacionadas con la percepción del apoyo social afectivo, así como entre la percepción del apoyo social de personas de confianza y las dimensiones de la vitalidad y la función social. Por el contrario, se encontró una relación inversa entre los promedios de los puntajes de las dimensiones de desempeño emocional, desempeño físico y salud general, y la percepción del apoyo social afectivo en la primera dimensión y de aquel de personas de confianza en las dos últimas.

Cada dimensión de la calidad de vida requirió ser ajustada según diferentes variables. La orientación sexual homosexual se asoció con un mejor puntaje en la dimensión de la función física, así como la escolaridad con una mejor puntuación en las dimensiones de bienestar emocional, función social y dolor corporal, y una menor en la dimensión del desempeño físico (cuadro 2). Todos los modelos se comportaron satisfactoriamente en relación con las suposiciones estadísticas para su validación.

\section{Discusión}

En el presente estudio, se analizó una muestra de personas de ambos sexos con HIV/sida inscritas en el programa de atención integral B24 de la Red Hospitalaria Méderi en Bogotá. Se valoraron los aspectos sociodemográficos y de la calidad de vida relacionados con la salud mediante el cuestionario SF-36 (26) y la percepción del apoyo social, mediante el DUKE-UNK-11 (19).

En la literatura médica se registra ampliamente información comprobada sobre el impacto que tiene la percepción del apoyo social en la calidad de vida de estos pacientes $(10,27)$. En dicho contexto, en el presente estudio se analizaron las posibles relaciones entre estos dos aspectos; una vez ajustados los efectos según las características sociodemográficas, se verificó la existencia de una relación significativa entre los puntajes observados en las escalas del cuestionario DUKE_UNC-11 y todos los dominios de la calidad de vida, lo cual concuerda con los hallazgos de otros estudios en la población colombiana, aunque en ellos se documentó dicha relación a partir de análisis bivariados $(28,29)$.

Los resultados evidenciaron la existencia de una relación directa entre el promedio de los puntajes en la dimensión del bienestar emocional y los dos dominios del apoyo social funcional, en tanto que las dimensiones de función física, desempeño emocional y dolor corporal, se relacionaron con la percepción del apoyo social afectivo $y$, las dimensiones de vitalidad y función social, con la percepción del apoyo social de personas de confianza.

Este hallazgo concuerda con estudios previos en otros países, en los cuales se han evidenciado asociaciones positivas significativas entre la percepción del apoyo social y puntuaciones más altas de la calidad de vida en aquellas personas con HIV/sida, quienes no presentan síntomas depresivos 0 de ansiedad ni perciben estar sometidas al estigma, y son funcionales en la resolución de problemas (30), por lo cual se adaptan mejor a vivir con una enfermedad crónica (29).

En el presente estudio también se encontró una relación inversa entre los promedios de los puntajes de las dimensiones del desempeño emocional y la percepción del apoyo social afectivo, asi como entre las dimensiones de desempeño físico y salud general y la dimensión del apoyo social de personas de confianza, en las dos últimas.

Esta situación, en principio contradictoria, se ha reportado en otros estudios, en los cuales las personas que habían recibido un apoyo social de personas de confianza (relacionado con apoyo financiero y asistencia material), se percibieron a sí 
mismas como más enfermas y con menor calidad de vida relacionada con la salud $(31,32)$. Otras hipótesis sugieren la mediación de circunstancias como la depresión, la pérdida del trabajo, la mala reacción a los medicamentos, el estigma o los estilos fallidos de afrontamiento, todos estos elementos comunes en las personas con HIV/sida (32).

La percepción del apoyo social es una variable mediadora fundamental en el proceso de estrés y se la considera como un elemento de resiliencia (9). Su evaluación proporciona información relevante en el curso de la enfermedad (14) y abre posibilidades de intervención orientadas a su control (9). Con base en los resultados del presente estudio, es posible sugerir que tanto el apoyo social de personas de confianza como el afectivo se comportan de manera independiente en su relación con las dimensiones de la calidad de vida, excepto en la dimensión del bienestar emocional, lo cual ha sido reportado también por otros autores (31-33).

Una vez incorporado el efecto del apoyo social funcional, el impacto de la variable del estado civil, en particular el estar divorciado, se asoció positivamente con el puntaje en el desempeño físico y la salud general, pero negativamente con los puntajes en la función física y la vitalidad. Este hallazgo puede explicarse por el hecho de que las personas con capacidades físicas disminuidas o con limitaciones en su actividad sexual, pueden requerir con mayor frecuencia apoyo para sus actividades privadas 0 de cuidado personal, las cuales no necesariamente son satisfechas por sus redes de amigos o conocidos, o en los ámbitos laborales o sociales (34). También, se ha reportado que la ausencia de asociación entre la pertenencia o no a una red de apoyo (asociaciones de pacientes con diagnóstico similar, por ejemplo) y las dimensiones de la calidad de vida, no necesariamente quiere decir que se suplan las necesidades sociales básicas de la persona (6). En concordancia con este hallazgo, Friedland, et al., han señalado que una mayor cantidad de personas con HIV en la red social reduce las potenciales fuentes de soporte (31).

Los resultados de este trabajo deben sopesarse con respecto a consideraciones metodológicas relevantes. En primer lugar, el tamaño de la muestra fue limitado, lo que afecta la capacidad de los métodos para captar con adecuada precisión las magnitudes de los efectos considerados, especialmente en grupos particularmente pequeños. En segundo lugar, el efecto mediador de algunas variables no se tomó en cuenta, por ejemplo, el tiempo que las personas habían mantenido un mismo estado civil en el momento de la encuesta. Tampoco se recolectó información sobre el desempeño clínico de los pacientes o sobre la duración del tratamiento con antirretrovirales, lo cual puede introducir confusión sobre las relaciones analizadas (35).

Por otro lado, la población incluida en este análisis hace parte de un programa en el que se ofrecen facilidades para la interacción social, el seguimiento del estado de salud y el cumplimiento de los tratamientos, sin que ello necesariamente invalide las conclusiones para personas con HIV/sida ajenas al programa. Además, el apoyo social se midió con un cuestionario no validado en la población colombiana, aunque en otras poblaciones latinoamericanas ha demostrado una adecuada concordancia interna (19), por lo que los hallazgos podrían servir para proponer hipótesis en otros estudios.

Pese a estas limitaciones, en el estudio se verificó la importancia del apoyo social funcional en el análisis de los niveles de la calidad de vida de las personas con HIV/sida y su comportamiento diferencial según los aspectos que se evalúen a partir de modelos multivariados. Además, se evidenció que, en ningún caso, el sexo de los sujetos o su edad parecen ser mediadores de importancia en esta relación.

En futuros estudios habrá que ahondar en la condición clínica de los sujetos, así como en la detección de situaciones que pueden afectar la adecuada percepción del apoyo social. Para esta población, sujeta al estigma derivado del contagio, el diagnóstico y los efectos en su comportamiento sexual (36), es necesario perfeccionar aspectos metodológicos que permitan comprender los conceptos de apoyo social y calidad de vida, así como su relación, especialmente cuando estas personas deben enfrentar, además, la precariedad económica, asistencial o laboral, lo que incide en el cumplimiento del tratamiento médico en los países en desarrollo (37).

En conclusión, los resultados proporcionan evidencia sobre las relaciones entre la calidad de vida relacionada con la salud y el apoyo social 
funcional percibido, así como entre la dimensión del bienestar emocional y los dos dominios del apoyo social funcional, en tanto que las dimensiones de la función física, el desempeño emocional y el dolor corporal se relacionaron con la percepción del apoyo social afectivo y las dimensiones de vitalidad y función social, con la percepción del apoyo social de personas de confianza.

Tomados en su conjunto, los hallazgos sugieren la necesidad de centrarse en las estrategias que fortalezcan las redes de apoyo social de estas personas y de incorporarlas en los planes de tratamiento de las instituciones de salud que brindan programas de atención integral. Las medidas reportadas por los cuestionarios SF36 y DUKE pueden ser útiles en la evaluación de los tratamientos médicos si se incorporan en el estudio variables como el análisis del impacto de la terapia antirretroviral en el estado de salud de estas personas.

\section{Agradecimientos}

A Laura Santana, pasante de la maestría en Salud Pública de la Universidad de Bordeaux (Francia), por su apoyo en la recolección de la información en la etapa inicial del proyecto. Al equipo del Programa B24 de Méderi, por facilitar la comunicación con las personas con HIV/sida, y a los encuestados, por su participación.

\section{Conflicto de intereses}

Los autores declaran que no existió ningún conflicto de intereses de tipo económico, comercial, familiar o laboral en el desarrollo del presente estudio.

\section{Financiación}

Este estudio no recibió financiación.

\section{Referencias}

1. Cardona-Arias J, Higuita-Gutiérrez L. Impact of HIV/AIDS on quality of life: Meta-analysis 2002-2012. Rev Esp Salud Pública. 2014;88:87-101. https://doi.org/10.4321/S1135-57 272014000100006

2. UNAIDS. The Gap Report. Geneva: Joint United Nations Programme on HIV/AIDS; 2014. Fecha de consulta: 5 de febrero de 2016. Disponible en: http://www.unaids.org/en/ resources/documents/2014/name,97466,en.asp

3. Cardona-Arias J, Peláez-Vanegas L, López-Saldarriaga $\mathbf{J}$, Duque-Molina M, Leal-Álvarez $\mathbf{O}$. Calidad de vida relacionada con la salud en adultos con $\mathrm{VIH} /$ sida, Medellín, Colombia, 2009. Biomédica. 2011;31:532-44. https://doi. org/10.7705/biomedica.v31i4.422

4. Su X, Lau JTF, Mak WW, Chen L, Choi KC, Song J, et al. Perceived discrimination, social support, and perceived stress among people living with HIV / AIDS in China. AIDS
Care. 2013;25:239-48. https://doi.org/10.1080/09540121.2 012.701713

5. Charkhian A, Fekrazad H, Sajadi H, Rahgozar M. Relationship between health-related quality of life and social support in HIV-infected people in Tehran, Iran. Iran J Public Health. 2014;43:100-6.

6. Bellón-Saameño J, Delgado-Sánchez A, Luna-del Castillo J de D, Lardelli-Claret P. Validez y fiabilidad del cuestionario de apoyo social funcional Duke-UNC-11. Aten Primaria. 1996;18:153-63.

7. Canaval E, Valencia G, Forero C, Guardela L, Magaña L, Vargas Y. Factores protectores y de riesgo para $\mathrm{VIH} /$ sida en mujeres de Cali, Colombia. Cienc Enferm. 2005;11:2333. https://doi.org/10.4067/S0717-95532005000200005

8. Wolf TM, Balson PM, Morse EV, Simon PM, Gaumer RH, Dralle PW, et al. Relationship of coping style to affective state and perceived social support in asymptomatic and symptomatic HIV-infected persons: Implications for clinical management. J Clin Psychiatry. 1991;52:171-3.

9. Remor E. Apoyo social y calidad de vida en la infección por el VIH. Aten Primaria. 2002;30:143-8. https://doi. org/10.1016/S0212-6567(02)78993-2

10. Bastardo Y, Kimberlin C. Relationship between quality of life, social support and disease-related factors in HIVinfected persons in Venezuela. AIDS Care. 2000;12:673-84. https://doi.org/10.1080/095401200750003842

11. Edwards L. Perceived social support and HIV/ AIDS medication adherence among African American women. Qual Heal Res. 2006;16:679-91. https://doi. org/10.1177/1049732305281597

12. Thoits PA. Conceptual, methodological, and theoretical problems in studying social support as a buffer against life stress. J Heal Soc Behav. 1982;23:145-59.

13. Leserman J, Petitto J, Golden R, Gaynes B. Impact of stressful life events, depression, social support, coping, and cortisol on progression to AIDS. Am J Psychiatry. 2000;157:1221-8. https://doi.org/10.1176/appi. ajp.157.8.1221

14. Chida Y, Vedhara K. Adverse psychosocial factors predict poorer prognosis in HIV disease: A meta-analytic review of prospective investigations. Brain Behav Immun. 2009;23:434-45. https://doi.org/10.1016/j.bbi.2009.01.013

15. Feeney BC, Collins NL. A new look at social support: A theoretical perspective on thriving through relationships. Pers Soc Psychol Rev. 2015;19:113-47. https://doi.org/10.1 $177 / 1088868314544222$

16. IndexMundi. VIH/SIDA - habitantes infectados con VIH/ SIDA por país - Mapa pomparativo de países - Mundo. Fecha de consulta: 5 de febrero de 2016. Disponible en: $\mathrm{http}: / / \mathrm{www}$.indexmundi.com/map/?v=35\&l=es

17. Méderi. Programa de atención integral de VIH - B24. Fecha de consulta: 16 de junio de 2016. Disponible en: http://www. mederi.com.co/index.php?option=com_content\&view=articl e\&id $=113 \& \mid$ temid $=115 \&$ showall $=1$

18. González A, Cañón V. VIH SIDA Nuevos paradigmas. Atención integral a personas que viven con el virus del VIH, Méderi. 2012. Fecha de consulta: 16 de junio de 2016. Disponible en: http://es.slideshare.net/nicorey2001/ programa-vih 
19. Piña LJ, Rivera IB. Validación del cuestionario de apoyo social funcional en personas seropositivas al VIH del noroeste de México. Cienc Enferm. 2007;XIl:53-63. https:// doi.org/10.4067/S0717-95532007000200007

20. Broadhead WE, Gehlbach SH, de Gruy FV, Kaplan BH. The Duke-UNC Functional Social Support Questionnaire. Measurement of social support in family medicine patients. Med Care. 1988;26:709-23.

21. Ware J, Sherbourne C. The MOS 36-item short-form health survey (SF-36): I. Conceptual framework and item selection. Med Care. 1992;30:473-83.

22. Ware JE, Snow KK, Kosinski M, Gandek B. SF-36 Health Survey Manual and Interpretation Guide. Boston, Massachusetts: New England Medical Center Hospitals; 1993.

23. Dadakis S, Yesalis CE, Bartels RH. Selection of regression models for health care data. Med Care. 2016;16:574-83.

24. Chatfield C. Model uncertainty, data mining and statistical inference. J R Stat Soc. 1995;158:419-66.

25. Breush T, Pagan A. A simple test for heteroscedasticity and random coefficient variation. Econometrica. 1979;47:128794.

26. Cardona J. Calidad de vida relacionada con la salud en personas con VIH/SIDA: comparación del MOSSF-36, WHOQOL-BREF y WHOQOL-HIV-BREF, Medellín, Colombia, 2009. Colomb Med. 2011;42:438-47.

27. Chambers L, Rueda S, Baker D, Wilson M, Deutsch R, Raeifar E, et al. Stigma, HIV and health: A qualitative synthesis. BMC Public Health. 2015;3:848. https://doi. org/10.1186/s12889-015-2197-0

28. Romero E, Armador C, Alvis L. Apoyo social y calidad de vida en pacientes con $\mathrm{VIH} / \mathrm{sida}$, residentes en Montería, Colombia. Revista Ciencias Médicas. 2010;1:173-9.

29. Vinaccia-Alpi S, Fernández H, Quiceno JM, LópezPosada M, Otalvaro C. Calidad de vida relacionada con la salud y apoyo social funcional en pacientes diagnosticados con VIH/Sida. Ter Psicol. 2008;26:125-32. https://doi. org/10.4067/S0718-48082008000100011

30. Prachakul W, Grant JS, Keltner NL. Relationships among functional social support, HIV-related stigma, social problem solving, and depressive symptoms in people living with HIV: A pilot study. J Assoc Nurses AIDS Care. 2007;18:67-76. https://doi.org/10.1016/j.jana.2007.08.002

31. Friedland J, Renwick R, Mccoll M. Coping and social support as determinants of quality of life in HIV/AIDS. AIDS Care. 1996;8:15-31. https://doi.org/10.1080/09540129650125966

32. Degroote S, Vogelaers D, Vandijck DM. What determines health-related quality of life among people living with HIV: An updated review of the literature. Arch Public Health. 2014;72:40. https://doi.org/10.1186/2049-3258-72-40

33. Burgoyne R, Renwick R. Social support and quality of life over time among adults living with HIV in the HAART era. Soc Sci Med. 2004;58:1353-66. https://doi.org/10.1016/ S0277-9536(03)00314-9

34. Centers for Disease Control and Prevention (CDC). Social support and health-related quality of life among older adults Missouri, 2000. MMWR Morb Mortal Wkly Rep. 2005 54:433-7.

35. Bajunirwe F, Tisch DJ, King CH, Arts EJ, Debanne SM, Sethi AK. Quality of life and social support among patients receiving antiretroviral therapy in Western Uganda. AIDS Care. 2009;21:271-9. https://doi.org/110.1080/09540120802 241863

36. Zallio V, Marconi L, Parenti P, Agostini M, Lupo S. Impacto del tratamiento antirretroviral en la función sexual de mujeres con VIH. Rev Méd Rosario. 2015;81:19-23.

37. De la Hoz J, Bolaño L, Cárdenas O, González R, Sabbag $\mathbf{J}$, Palacio $\mathbf{L}$, et al. Characterization of treatment failure in HIV positive patients in the Colombian Caribbean region. Colomb Med. 2014;45:162-8. 